\title{
Morphological and functional alterations of the intestine of rats with iron-deficiency anemia
}

M.L.C. Wayhs ${ }^{1}$, F.S.R. Patrício ${ }^{2}$, O.M.S. Amancio ${ }^{1}$, M.Z. Pedroso ${ }^{1}$, U. Fagundes Neto ${ }^{1}$ and M.B. Morais ${ }^{1}$
${ }^{1}$ Disciplina de Gastroenterologia Pediátrica, and '2Departamento de Patologia, Universidade Federal de São Paulo, Escola Paulista de Medicina, São Paulo, SP, Brasil

\section{Correspondence \\ M.B. Morais \\ Rua Araioses, 75 \\ 05442-010 São Paulo, SP \\ Brasil \\ Fax: +55-11-5549-6124 \\ E-mail: mbmorais@osite.com.br}

Research supported by CNPq.

Publication supported by FAPESP.

Received October 10, 2003

Accepted July 28, 2004

\section{Abstract}

The present study was designed to assess the intestinal absorption of D-xylose and jejunal morphometry in rats with iron-deficiency anemia. Male Wistar rats were randomly divided into a control group (diet containing $50 \mathrm{mg} \mathrm{Fe} / \mathrm{kg}, \mathrm{N}=12$ ) and an anemic group (diet containing $<5 \mathrm{mg} \mathrm{Fe} / \mathrm{kg}, \mathrm{N}=12$ ). The animals were housed in individual metabolic cages and deionized water and diet were provided ad libitum for 6 weeks. Hemoglobin and hematocrit were determined at 0 , 2,4 , and 6 weeks. At the end of the study the rats were submitted to a D-xylose absorption test $(50 \mathrm{mg} / 100 \mathrm{~g}$ body weight) and sacrificed and a jejunal specimen was obtained for morphometric study. At the end of the study the hemoglobin and hematocrit of the anemic rats (8.7 $\pm 0.9 \mathrm{~g} / \mathrm{dl}$ and $34.1 \pm 2.9 \%$, respectively) were significantly $(\mathrm{P}<0.05)$ lower than those of the controls $(13.9 \pm 1.4 \mathrm{~g} / \mathrm{dl}$ and $47.1 \pm 1.5 \%$, respectively). There was no statistical difference in D-xylose absorption between the anemic $(46.5 \pm 7.4 \%)$ and control $(43.4 \pm 9.0 \%)$ groups. The anemic animals presented statistically greater villus height $(445.3 \pm 36.8 \mu \mathrm{m})$, mucosal thickness $(614.3 \pm 56.3 \mu \mathrm{m})$ and epithelial surface $(5063.0 \pm 658.6 \mu \mathrm{m})$ than control $(371.8 \pm 34.3,526.7 \pm 62.3$ and $4401.2 \pm 704.4 \mu \mathrm{m}$, respectively; $\mathrm{P}<0.05)$. The increase in jejunum villus height, mucosal thickness and epithelial surface in rats with iron-deficiency anemia suggests a compensatory intestinal mechanism to increase intestinal iron absorption.

\section{Introduction}

Iron plays a significant role in several metabolic processes, and iron balance is very important because both iron excess and deficiency are deleterious. The small intestine is the main organ responsible for the homeostasis of iron by controlling its absorption in response to changes in the amount of iron in
Key words - Iron deficiency

- Anemia

- Jejunal villus height

- Jejunal mucosal thickness

- Jejunal epithelial surface the body. Iron deficiency directly influences the intestinal capacity of iron absorption. In iron deficiency there is an increase in the expression of proteins for iron absorption in the small intestine, such as divalent metal transport 1, mobilferrin, ferroportin-1, ceruloplasmin, and hepaestin (1-3). Iron deficiency may also cause modification in intestinal morphology and function including vil- 
lus atrophy $(4,5)$, reduced intestinal absorption of D-xylose $(4,5)$, decrease in intestinal disaccharidase (6), and alteration in intestinal permeability $(7,8)$. Administration of lactulose and rhamnose before and after iron therapy to otherwise healthy children with iron-deficiency anemia (7) demonstrated that the lactulose/rhamnose ratio was significantly higher in the iron-deficient state than after iron therapy. This result is consistent with studies which demonstrated impaired xylose absorption and varying degrees of small intestinal mucosal atrophy in anemic children that was corrected with iron therapy $(4,5)$. Previous studies did not detect villus atrophy in the intestinal mucosa of rats with irondeficiency anemia, as reported for humans $(9,10)$. Taking into account that abnormalities in intestinal permeability and villus atrophy can be nonspecific, the possible influence of environmental factors besides iron deficiency anemia in human studies performed in underdeveloped countries $(5,8)$ should be considered since these intestinal abnormalities may be the consequence of tropical or environmental enteropathy $(11,12)$.

Therefore, the objective of the present experimental study conducted on laboratory animals was to evaluate the intestinal absorption of D-xylose and the jejunal morphometry of rats with iron-deficiency anemia under controlled environmental conditions.

\section{Material and Methods}

Male Wistar rats 21 days of age were randomly divided into a control group $(\mathrm{N}=$ 12) and an anemic group $(\mathrm{N}=12)$. The control group received the AIN 93-G diet containing $50 \mathrm{mg}$ elemental iron per $\mathrm{kg} \operatorname{diet}(13,14)$. The anemic group was fed the same diet, but containing less than $5 \mathrm{mg}$ elemental iron per $\mathrm{kg}$ diet. The rats were housed in acrylic and stainless steel metabolic cages (metabolic cage 650-0100; Nalgene, Rochester, NY, USA) in a room with a 12 -h light-dark cycle.
Weight was used as a growth indicator. Feed efficiency was calculated by dividing weight gain by food consumption during the study period (13). Hemoglobin and hematocrit were determined at $0,2,4$, and 6 weeks. Blood samples were obtained from a tail vein. Hematocrit was measured by centrifugation of blood collected into heparinized microcapillary tubes. Hemoglobin concentration was measured by the cyanmethemoglobin method.

D-xylose absorption test was performed at the end of the 6-week study. A 10\% aqueous solution of D-xylose $(50 \mathrm{mg} / 100 \mathrm{~g}$ body weight) was infused by gavage into the stomach and urine collection was started right after D-xylose administration. The volume of urine collected for $5 \mathrm{~h}$ was carefully measured and the amount of D-xylose in urine was determined (15).

At the end of the study the rats were anesthetized, the abdominal cavity was opened and a jejunum specimen about $2 \mathrm{~cm}$ long was taken distally just below the ligament of Treitz. This jejunum specimen was fixed in formaldehyde, embedded in paraffin and stained with hematoxylin and eosin. The morphometric study was performed using the Image Pro-plus 3.0 (Media Cybernetics, Silver Spring, MD, USA) image analyzer software, which permitted the following measurements: 1) villus height (distance from the tip of the villus to the villus-crypt junction); 2) total mucosal thickness (distance from the tip of the villus to the muscularis mucosae); 3) crypt depth (difference between total mucosal thickness and villus height); 4) villus/crypt ratio; 5) surface length (contour of the luminal border of the epithelial surface in a 1-mm muscularis mucosae field); 6) enterocyte height (distance between the basal membrane and the luminal surface of the enterocyte). For each rat, villus height, total mucosal thickness and crypt depth were measured at 15 sites of the jejunum specimen and surface length was measured at two sites. The height of 20 enterocytes was meas- 
ured in the middle third of the villus.

The rats were killed by exsanguination from the abdominal aorta and the liver was extracted for the determination of its iron content. After obtaining the dry weight, the liver was submitted to acid digestion and the iron content was determined by flame atomic absorption spectrophotometry (5.100; PerkinElmer, Norwalk, CT, USA) (16).

Statistical analysis was performed using the Sigma Stat software, version 2.0. Data are reported as means $+\mathrm{SD}$. The Student $t$ test was used to compare the means of the variables of the iron deficiency group with the control group.

The study was conducted with the approval of the Ethics Committee in Research of the Federal University of São Paulo, Escola Paulista de Medicina, São Paulo, SP, Brazil.

\section{Results}

There were no statistically significant differences in hemoglobin or hematocrit between the animals in the anemic and control groups at the beginning of the experiment (Table 1). From the second week onwards, the hemoglobin and hematocrit of the rats in the anemic group were significantly lower than those of the control group. The mean hepatic iron of the anemic group was also significantly lower than that of the control group (Table 1). Initially, the mean body weight of the rats in the anemic group was similar to the mean of the control group. After six weeks of dietary treatment, the mean body weight of the anemic group (223.3 $\pm 42.5 \mathrm{~g}$ ) was statistically lower than that of the control group $(254.7 \pm 31.2 ; \mathrm{P}=0.026)$. The daily mean food intake by the control group (12.2 $\pm 1.8 \mathrm{~g})$ was statistically greater than the intake by the anemic group (14.0 \pm $1.4 \mathrm{~g} ; \mathrm{P}=0.016$ ), but the groups did not differ significantly in terms of feed efficiency (g weight gained/g food intake: 0.38 \pm 0.04 and $0.39 \pm 0.03$ for anemic and control animals, respectively.
After 6 weeks of dietary treatment, the mean percent urinary excretion of D-xylose of $46.5 \pm 7.4 \%$ by the anemic group was not statistically different from $43.4 \pm 9.0 \%$ by the control group.

Regarding jejunal morphometry, the anemic rats had a significantly greater villus height, total mucosal thickness and surface length than the controls. However, there was no significant difference between groups in crypt depth, villus/crypt ratio or enterocyte height (Table 2).

Table 1. Hemoglobin, hematocrit and hepatic iron in rats with iron-deficiency anemia.

\begin{tabular}{lcc}
\hline & $\begin{array}{c}\text { Anemic } \\
\text { group }\end{array}$ & $\begin{array}{c}\text { Control } \\
\text { group }\end{array}$ \\
\hline Hemoglobin (g/dl) & $11.2 \pm 0.9$ & $11.0 \pm 0.7$ \\
Baseline & $7.4 \pm 1.1^{*}$ & $13.3 \pm 1.5$ \\
2 weeks on diet & $8.1 \pm 1.3^{*}$ & $13.7 \pm 2.1$ \\
4 weeks on diet & $8.7 \pm 0.9^{*}$ & $13.9 \pm 1.4$ \\
6 weeks on diet & & \\
& & \\
Hematocrit (\%) & $35.8 \pm 3.4$ & $35.1 \pm 3.9$ \\
Baseline & $28.1 \pm 2.8^{*}$ & $43.4 \pm 2.7$ \\
2 weeks on diet & $30.8 \pm 2.8^{*}$ & $46.4 \pm 1.3$ \\
4 weeks on diet & $34.1 \pm 2.9 *$ & $47.1 \pm 1.5$ \\
6 weeks on diet & & \\
Hepatic iron (mg/g) & & \\
6 weeks on diet & $0.15 \pm 0.09^{*}$ & $0.40 \pm 0.16$ \\
\hline Anemic rats received an AlN 93-G diet containing \\
5 mg elemental iron/kg diet and controls received \\
the same diet containing 50 mg/kg diet. Data are \\
reported as means \pm SD for 12 rats in each group. \\
* $<$ 0.05 compared to control rats (Student $t$ - \\
test).
\end{tabular}

Table 2. Jejunal morphometry of rats with iron-deficiency anemia.

\begin{tabular}{lcc}
\hline & Anemic rats & Control rats \\
\hline Villus height $(\mu \mathrm{m})$ & $445.3 \pm 36.8^{*}$ & $371.8 \pm 34.30$ \\
Crypt depth $(\mu \mathrm{m})$ & $169.0 \pm 38.9$ & $154.9 \pm 41.2$ \\
Villus/crypt ratio & $2.9 \pm 0.8$ & $2.6 \pm 0.6$ \\
Total mucosal thickness $(\mu \mathrm{m})$ & $614.3 \pm 56.3^{*}$ & $526.7 \pm 62.3$ \\
Surface length $(\mu \mathrm{m})$ & $5063.0 \pm 658.6^{*}$ & $4401.2 \pm 704.4$ \\
Enterocyte height $(\mu \mathrm{m})$ & $30.6 \pm 1.4$ & $30.3 \pm 2.3$ \\
\hline
\end{tabular}

Anemic rats received an AIN 93-G diet containing $5 \mathrm{mg}$ elemental iron $/ \mathrm{kg}$ diet and controls received the same diet containing $50 \mathrm{mg} / \mathrm{kg}$ diet. Data are reported as means \pm SD for 12 rats in each group.

${ }^{*} \mathrm{P}<0.05$ compared to control rats (Student $t$-test). 


\section{Discussion}

The objective of the present study was to evaluate the jejunal morphometry of rats with iron-deficiency anemia in order to avoid the influence of environmental factors, which may affect $\mathrm{D}$-xylose absorption by the intestine and intestinal morphology in studies conducted on human beings. The iron-deficiency anemia group of rats presented greater villus height, total mucosal thickness and intestinal surface extension compared to control (Table 2). Previous studies $(9,10)$ did not find changes in the intestinal histology of rats with anemia. Hence, in a situation in which it was possible to control the environmental conditions using laboratory animals, iron-deficiency anemia did not determine villus atrophy in the small intestine of rats as observed in children $(4,5)$. This finding may be evidence that in humans the intestinal response to iron deficiency may be inhibited by some other factor with a stronger effect on intestinal morphology and function as observed in environmental or tropical enteropathy $(11,12)$.

This hypothesis is in agreement with the profile of D-xylose absorption, which is absorbed in the duodenum and jejunum, mainly by passive absorption without being modified. D-xylose has been used as an indicator of villus atrophy in the small intestine (17). In clinical studies, when villus atrophy is present a reduced absorption of D-xylose also occurs, both in children and in adults with iron-deficiency anemia (4-6). However, our results did not show any difference in the intestinal absorption of D-xylose based on urinary excretion between the anemic group and the control, which confirms the results of a similar study in rats (18). Further evidence of a difference between clinical and experimental studies comes from the evaluation of intestinal permeability to lactulose/ mannitol or rhamnose. Studies involving children in Israel (7) and Guatemala (8) demonstrated a higher ratio of lactulose/manni- tol or rhamnose in children with iron deficiency, suggesting a decrease of the intestinal surface and/or increase of the lactulose passage through the paracellular space. In contrast, a decrease of the lactulose/mannitol ratio was reported in a study on rats with iron-deficiency anemia (19), compatible with the increase of villus height and of intestinal surface length, which were the most important findings of the present study.

Partial villus atrophy and decreased Dxylose absorption demonstrated both in children and in adults with iron-deficiency anemia (4-6) may be related, at least in part, to environmental influences and be a consequence of environmental enteropathy, which determines this nonspecific, functional and morphological abnormalities of the small intestine $(11,12)$. Therefore, under controlled laboratory conditions using laboratory animals, iron-deficiency anemia did not determine villus atrophy in the small intestine as previously found in children with iron deficiency anemia $(4,5)$.

The meaning of the increased villus height and intestinal surface may be interpreted, at least in part, by considering the intestinal adaptation to iron deficiency. Smith et al. (20) studied the structural and cellular adaptation of duodenal cells in adult rats receiving an iron-deficient diet and observed an increase in iron uptake by the enterocytes in the middle third of the villus associated with lengthening and broadening of the duodenal villi. Similar results were observed in a study in which mice with and without the hemochromatosis genotype received iron-deficient, normal and iron-rich diets. Normal mice that received the iron-deficient diet presented an increase of villus height and of ferric reductase activity (21). In our study, the mean hemoglobin of the group with irondeficient anemia corresponded to approximately $60 \%$ of the mean hemoglobin of the control group, indicating that the increase in villus height was also present in rats in which the iron-poor diet was administered since 
weaning and was causing a more severe iron-deficiency anemia. This aspect differentiates our study from those published by Smith et al. (20) and Simpson et al. (21) who used animals with mild iron deficiency.

In conclusion, contrary to what was observed in studies with humans, in which iron-deficiency anemia is associated with a decrease in D-xylose absorption and with partial atrophy of intestinal villi, the computerized morphometry used in our study allowed us to show lengthening of the intestinal villi in growing rats with severe irondeficiency anemia. This finding may be explained, at least in part, as a compensatory intestinal mechanism to increase intestinal iron absorption.

\section{References}

1. Morgan E \& Oates $P$ (2002). Mechanisms and regulation of intestinal iron absorption. Blood Cells, Molecules, and Diseases, 29: $384-$ 399.

2. Zoller H, Theurl I, Koch R, McKie A, Vogel W \& Weiss G (2003). Duodenal cytochrome $\mathrm{b}$ and hepaestin expression in patients with iron deficiency and hemochromatosis. Gastroenterology, 125: 746754.

3. Simovich M, Hainsworth L, Fields P, Umbreit J \& Conrad M (2003). Localization of the iron transport proteins mobilferrin and DMT-1 in the duodenum: the surprising role of mucin. American Journal of Hematology, 74: 32-45.

4. Naiman JL, Oski FA, Diamond LK, Vawter GF \& Shwachman H (1964). The gastrointestinal effects of iron-deficiency anemia. Pediatrics, 33: 83-99.

5. Guha DK, Walia BNS, Tandon BN, Deo MG \& Ghai OP (1968). Small bowel changes in iron-deficiency anaemia of childhood. Archives of Diseases in Childhood, 43: 239-244.

6. Prassad A \& Prassad C (1991). Iron deficiency: non-hematological manifestations. Progress in Food and Nutrition Science, 15: 255283.

7. Berant M, Khourie M \& Menzies IS (1992). Effect of iron deficiency on small intestinal permeability in infants and young children. Journal of Pediatric Gastroenterology and Nutrition, 14: 17-20.

8. Goto K, Chew F, Torún B, Peerson JM \& Brown KH (1999). Epidemiology of altered intestinal permeability to lactulose and mannitol in Guatemalan infants. Journal of Pediatric Gastroenterology and Nutrition, 28: 282-290

9. Valberg LS, Taylor KB \& Witts LJ (1961). The effect of iron deficiency on the stomach of the rat. British Journal of Nutrition, 15: 473-480.

10. Buts J-P, Delacroix DL, Dekeyser N, Paquet S, Horsmans $Y$, Boelens M, Van Craynest MP \& De Meyer R (1984). Role of dietary iron in maturation of rat small intestine at weaning. American Journal of Physiology, 246: G725-G731.

11. Fagundes-Neto U, Viaro T, Wehba J, Patricio FSR \& Machado NL
(1984). Tropical enteropathy (environmental enteropathy) in early childhood: a syndrome caused by contaminated environment. Journal of Tropical Pediatrics, 30: 204-209.

12. Menzies IS, Zuckerman MJ, Nukajam WS, Somasundaram SG, Murphy B, Jenkins AP, Crane RS \& Gregory GG (1999). Geography of intestinal permeability and absorption. Gut, 44: 483-489.

13. Beard JL, Zhan CS \& Brigham DE (1995). Growth in iron-deficient rats. Proceedings of the Society for Experimental Biology and Medicine, 209: 65-72.

14. Reeves PG, Nielsen FH \& Fahey Jr GC (1993). AIN-93 purified diets for laboratory rodents: final report of the American Institute of Nutrition ad hoc writing committee on the reformulation of the AIN76 A rodent diet. Journal of Nutrition, 123: 1939-1951.

15. Eberts TJ, Sample RHB, Glick MR \& Ellis GH (1979). A simplified, colorimetric micromethod for xylose in serum or urine, with phloroglucinol. Clinical Chemistry, 25: 1440-1443.

16. Marks GE, Moore CE, Kanabrocki EL, Oester YT \& Kaplan E (1971). Determination of trace elements in human tissue. I. Cd, Fe, $\mathrm{Zn}, \mathrm{Mg}$ and Ca. Applied Spectroscopy, 26: 523-527.

17. Craig RM \& Ehrenpreis ED (1999). D-xylose testing. Journal of Clinical Gastroenterology, 29: 143-150.

18. Sharma DC, Singh PP \& Simlot MM (1973). Small intestinal absorptive functions in iron deficient rats. Digestive Diseases, 18: 73-74.

19. Morais MB \& Lifschitz CH (2004). Intestinal permeability to lactulose and mannitol in growing rats with iron deficiency anemia. Biological Trace Element Research, 99: 233-240.

20. Smith MW, Debnam ES, Dashwood MR \& Srai SK (2000). Structural and cellular adaptation of duodenal iron uptake in rats maintained on an iron-deficient diet. Pflügers Archiv. European Journal of Physiology, 439: 449-454.

21. Simpson R, Debnam E, Beaumont N, Bahram S, Schumann K \& Srai $S$ (2003). Duodenal mucosal reductase in wild-type and Hfe knockout mice on iron adequate, iron deficient, and iron rich feeding. Gut, 52: $510-513$. 